\title{
Optimización y clasificación de señales EMG a través de métodos de reconocimiento de patrones
}

\author{
Optimization and Classification of EMG Signals Through \\ Pattern Recognition Methods
}

\author{
Cristhian Manuel Durán Acevedo \\ PhD. en Ingeniería Electrónica, URV-UPC \\ Sistemas Multisensoriales y Reconocimiento de Patrones. \\ Automatización y Control. \\ Hardware DSP \\ Docente Tiempo Completo, Investigador Grupo GISM \\ Universidad de Pamplona \\ Pamplona, Colombia \\ cristhianmanuel@gmail.com
}

\author{
Aylen Lisset Jaimes Mogollón \\ M. Sc(c) Controles Industriales, Universidad de Pamplona. \\ Docente Cátedra, Investigador Grupo GISM, \\ Universidad de Pamplona \\ Pamplona, Colombia \\ lissetjaimes@gmail.com
}

Resumen-Este artículo presenta un estudio basado en la optimización de la respuesta de un electromiógrafo a través de algunas técnicas utilizadas para el tratamiento, análisis y procesamiento de señales electromiográficas superficiales, con el fin de proporcionar una herramienta útil como estrategia para el diagnóstico y pronóstico de cuadro clínico de enfermedades musculares (Por ejemplo, para pacientes con pie caído).

Los datos fueron obtenidos a partir de la información de pacientes que fueron diagnosticados previamente por fisiatras, de los cuales 7 eran sanos y 5 pacientes mostraron neuropatía de pie caído.

Un conjunto de señales electromiográficas fueron adquiridas y almacenadas durante el movimiento de dorsiflexión en una posición supina a partir del músculo tibial anterior en cada paciente. Luego estas señales fueron procesadas mediante técnicas de extracción característica y métodos de reconocimiento de patrones para la clasificación de las mismas.

Para el pre-procesamiento de las señales electromiográficas se emplearon métodos en tiempo y frecuencia, como la transformada de Fourier y, a su vez, técnicas como el Análisis de Componentes Principales (PCA) y redes neuronales artificiales (es decir, MLP y PNN), que permiten representar en forma gráfica los resultados obtenidos en un plano bidimensional y mejorar de esta forma el porcentaje de clasificación.

Los resultados obtenidos describen un sistema electromiógrafo, el cual fue optimizado mediante la implementación de métodos de reconocimiento de patrones, se alcanzó un porcentaje de acierto de hasta el $100 \%$ en la clasificación de señales EMG por medio de electrodos superficiales.

Palabras clave— EMG, FFT, MLP, PCA, pie caído, PNN.

\begin{abstract}
This paper presents a study based on the response optimization of an electromyograph through processing techniques for the analysis of surface electromyographic signals, in order to provide a useful tool as a strategy for the diagnosis and prognosis of clinical symptoms of muscle diseases (e.g. for patients with foot drop). The patients were previously diagnosed by physiatrists, seven of them were healthy and five showed foot drop neuropathy. A set of electromyographic signals were acquired and stored during the movement of dorsiflexion in the supine position from the tibialis anterior muscle in each of patients. Then, these signals were processed with feature extraction techniques and pattern recognition methods for their classification.
\end{abstract}

Regarding the data preprocessing of electromyographic signals, methods of time and frequency such as Fourier Transform and Principal Component Analysis (PCA) and Artificial Neural Networks (i.e. MLP and PNN) were used to represent graphically in a two-dimensional plane the results obtained and thus to improve the classification percentage. The results describe an electromyography, which was optimized with pattern recognition methods, achieving a success rate of $100 \%$ in the classification of EMG signals, via surface electrodes.

Keywords- EMG, FFT, MLP, PCA, foot drop, PNN.

\section{INTRODUCCIÓN}

La electromiografía es una metodología de registro y análisis de la actividad bioeléctrica del músculo esquelético, orientada al diagnóstico de las enfermedades neuromusculares. Estos sistemas han evolucionado paralelamente al conocimiento de las propiedades de la energía eléctrica 
y al desarrollo de la tecnología eléctrica y electrónica. A mediados del siglo XX se introdujo el primer equipo comercial de electromiografía para uso médico, basado en circuitos electrónicos analógicos. El desarrollo posterior de la tecnología digital ha permitido disponer de sistemas expertos controlados por microprocesadores, cada vez, más fiables y potentes para captar, representar, almacenar, analizar y clasificar las señales mioeléctricas [1]. El avance de las nuevas tecnologías de la información y la comunicación puede conducir en un futuro próximo a la aplicación de desarrollos de inteligencia artificial que faciliten la clasificación automática de señales así como sistemas expertos de apoyo al diagnóstico electromiográfico [2].

El objetivo del presente artículo es optimizar la respuesta de un electromiógrafo con electrodos de superficie, utilizado en pacientes con pie caído a través de técnicas de procesamiento de datos y métodos de reconocimiento de patrones para la clasificación de medidas.

\section{A. Concepto de Unidades Motoras}

Las unidades motoras ó motoneuronas son las encargadas de transmitir los impulsos nerviosos y llevarlos hasta el músculo, las cuales son controladas, a su vez, por centros nerviosos superiores que regulan la respuesta motriz.

Los axones de las motoneuronas parten desde la medula espinal y llegan hasta las fibras musculares. Cada axón poco antes de conectar con estas fibras se divide y ramifica en muchos terminales, cada uno de los cuales contacta con una fibra a través de una estructura llamada "Placa Motora "[3].

En la Fig. 1 se observa al conjunto formado por una motoneurona y las fibras musculares que inerva se le llama "Unidad Motora" (U.M).

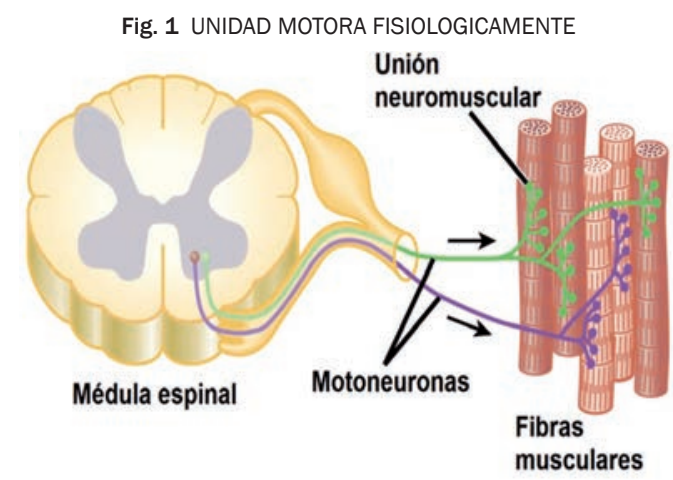

Fuente: [Online]. Available http://www.pucpr.edu/marc/facultad/asantiago/anatomia/Muscular.htm\#slide0033.htm

\section{B. Reclutamiento de las unidades motoras}

1) Reclutamiento espacial

Se aplica por la ley de Henneman (1965). El reclutamiento está ordenado por el principio del tamaño o "size principle". Las primeras motoneuronas reclutadas son las motoneuronas de tamaño pequeño. Son las que tienen igualmente la velocidad de conducción más débil y que desarrollarán la tensión muscular más baja [4] y [5].

\section{2) Reclutamiento temporal}

Se trata de la suma temporal: un músculo responde mediante una sacudida a un impulso, si un siguiente impulso surgiera suficientemente rápido, la sacudida sería superior (hay suma).

\section{MÉTODOS EXPERIMENTALES}

La Fig. 2. muestra un diagrama en bloques estructurado de tal manera que se puede seguir un orden y un análisis desde el músculo del paciente hasta el tratamiento de la señal.

Está compuesto por:

- Captación de la señal (sensores).

- Acondicionamiento (amplificadores).

- Conversor análogo digital (tarjeta de adquisición de datos)

- Procesamiento (PC).

Fig. 2 DIAGRAMA EN BLOQUES DEL SISTEMA

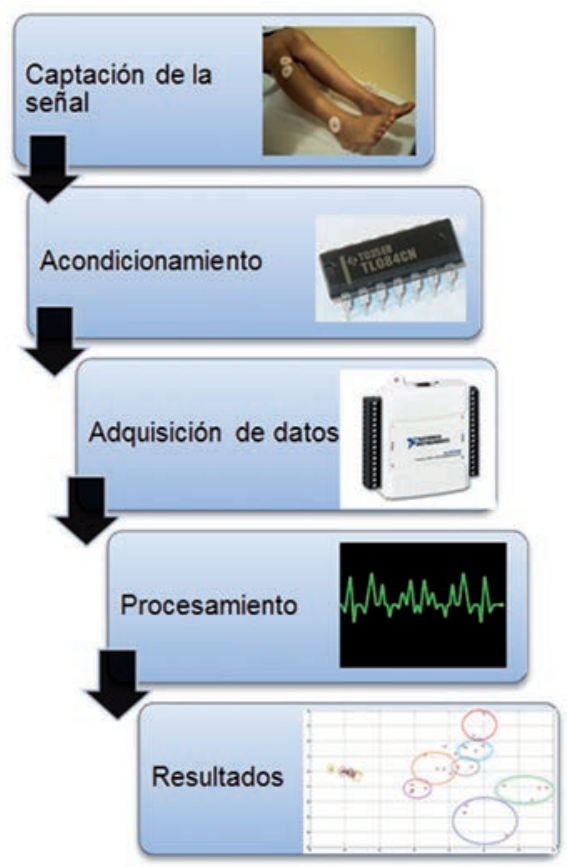

Fuente: Autores del proyecto 


\section{A. Muestras}

El conjunto de medidas realizadas fueron obtenidas a partir de una muestra de 12 personas, con el apoyo y valoración de los Fisiatras: Dr. Ómar Albarracín Acosta, Universidad de Pamplona y el Dr. Larry Rubio de la Fundación Médico Preventiva-Pamplona, previamente tratados y diagnosticados en estas entidades.

En la Tabla I, se muestran algunas de sus características.

TABLA I.

CARACTERISTICAS DE LOS PACIENTES REGISTRADOS

\begin{tabular}{|c|c|c|c|c|c|c|}
\hline 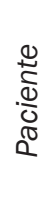 & 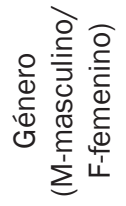 & 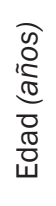 & \begin{tabular}{l} 
Do \\
\multirow{2}{*}{} \\
0 \\
0 \\
0 \\
0
\end{tabular} & 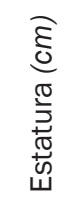 & 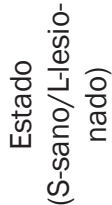 & : \\
\hline$A$ & $M$ & 36 & 75 & 170 & $S$ & \\
\hline$B$ & $M$ & 32 & 80 & 180 & $S$ & \\
\hline$C$ & $M$ & 23 & 70 & 170 & $S$ & \\
\hline$D$ & $M$ & 12 & 42 & 130 & $S$ & \\
\hline$E$ & $\mathrm{~F}$ & 22 & 58 & 165 & $S$ & \\
\hline$F$ & $\mathrm{~F}$ & 19 & 50 & 159 & $S$ & \\
\hline$G$ & $\mathrm{M}$ & 12 & 43 & 145 & $S$ & \\
\hline 1 & M & 37 & 69 & 166 & $L$ & 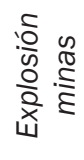 \\
\hline 2 & $\mathrm{~F}$ & 40 & 62 & 164 & $L$ & $\begin{array}{ll}0 & 0 \\
0 & \frac{\pi}{2} \\
\frac{\pi}{0} & \frac{0}{\pi} \\
\frac{0}{\pi} & 0 \\
0 & 0\end{array}$ \\
\hline 3 & $\mathrm{~F}$ & 49 & 58 & 160 & $L$ & 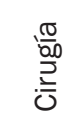 \\
\hline 4 & $\mathrm{~F}$ & 73 & 75 & 157 & $L$ & $\frac{\pi}{\frac{\pi}{\pi}}$ \\
\hline 5 & M & 63 & 63 & 156 & $L$ & 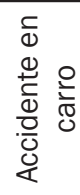 \\
\hline
\end{tabular}

Fuente: Autor del proyecto

\section{B. Materiales}

Se registró la actividad del músculo tibial anterior proveniente de contracciones isométricas; habida cuenta que la MCV es la mayor fuerza que es capaz de desarrollar el sistema nervioso y muscular. Se indicó a los pacientes realizar la mayor fuerza de forma voluntaria, durante un tiempo de cuatro segundos, digitalizadas a una frecuencia de muestreo (fs) de 10 kHz con el "Sistema Registrador Electromiográfico de Superficie”, ver Fig. 3 [6].

Se utilizaron electrodos superficiales ubicados según normativa SENIAM (Surface ElectroMyoGraphy for the Non-Invasive Assessment of Muscles), se indica que deben ser colocados a 1/3 en la línea entre la punta del peroné y la punta del maléolo medial, mostrado en la Fig. 4 [7].

FIG. 3 SISTEMA REGISTRADOR ELECTROMIOGRÁFICO DE SUPERFICIE

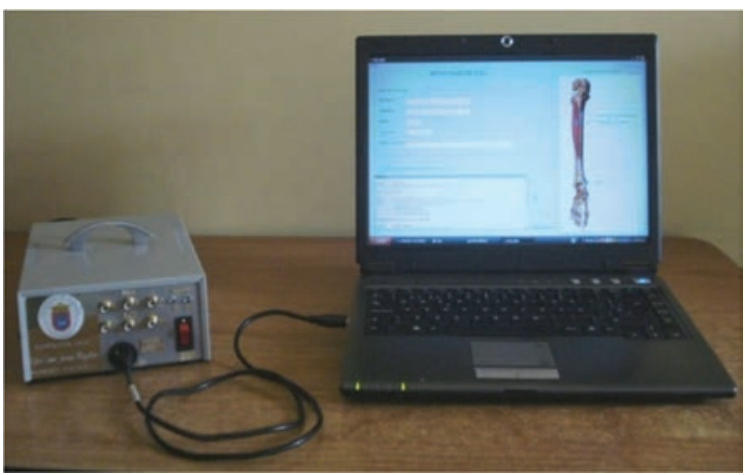

Fuente: Autores del proyecto

En la Tabla II se describen los parámetros entre los cuales depende en gran medida el funcionamiento del equipo EMG.

TABLA II.

PARÁMETROS REGISTRADOR EMG

\begin{tabular}{|c|c|}
\hline Rango de Frecuencia & $10-500 \mathrm{~Hz}$ \\
\hline CMRR & $120 \mathrm{~dB}$ \\
\hline Rango de Ruido & $0-20 \mathrm{~Hz}$ \\
\hline Rango de Amplitud & $100 \mu \mathrm{V}-20 \mathrm{mV}$ \\
\hline Ganancia total & 1001 \\
\hline
\end{tabular}

Fuente: Autores del proyecto 


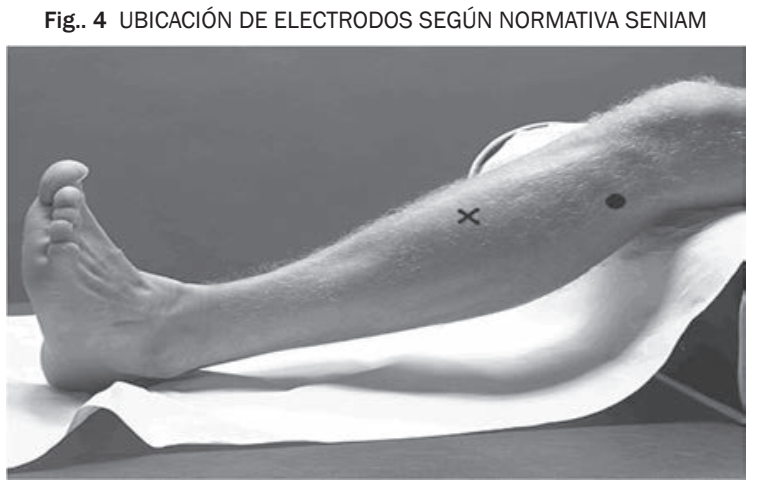

Fuente: [Online]. Available: http://www.seniam.org

La señal fue registrada en un ordenador, donde fue monitoreada constantemente y luego almacenada para posteriormente realizar el análisis respectivo. El software utilizado para la adquisición y análisis de datos fue el Matlab R2009a.

\section{Protocolo de medición}

En todos los pacientes se siguió el mismo protocolo de medición. Para la adquisición de cada una de las muestras se emplearon las siguientes pautas:

1) No se aplicaron lociones ni cremas sobre el área de análisis un día antes de realizar la prueba.

2) Se acomodaron en una posición supina.

3) La zona donde se localizaron los electrodos estaba debidamente depilada.

4) Se limpió la piel con alcohol antiséptico.

5) A continuación se localizaron los electrodos.

6) Se finalizó con el test Clínico.

En total se adquirieron 60 muestras y por cada paciente se realizaron 5 repeticiones.

\section{Tratamiento de los datos}

\section{1) Pre-procesamiento}

El pre-procesamiento prepara los datos para su extracción. La toma de datos del sensor implica una entrada de información innecesaria que hace lento el procesamiento y dificulta la extracción, en esta sección se hace una primera selección de la información al eliminar componentes de ruido principalmente.

\section{2) Centralización de datos}

Ajusta las señales a una escala adecuada.

En las Fig. 5 y Fig. 6 se observan las señales obtenidas al haber aplicado la técnica de centrado de datos, el cual es una normalización donde a cada una de las columnas se le resta su valor medio. Es decir, toda medida es descrita por variables de media nula.

Fig.. 5 SEÑAL ORIGINAL SIN NORMALIZAR

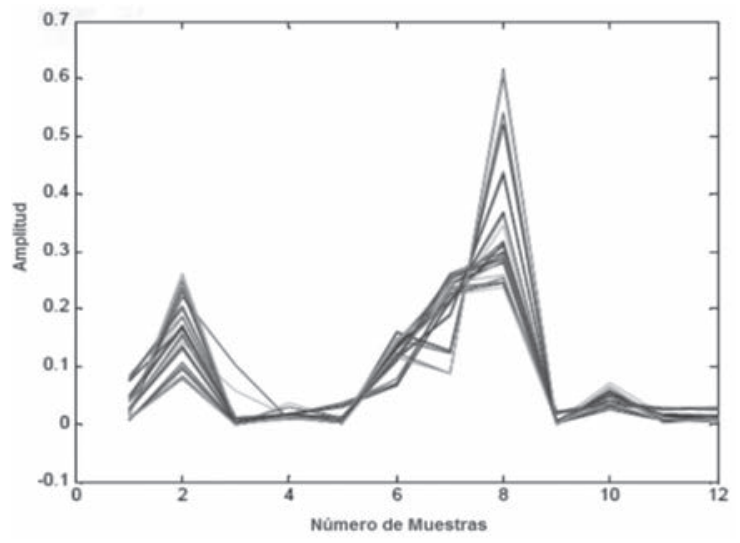

Fuente: Autores del proyecto

Fig. 6 SEÑAL CON CENTRADO DE DATOS

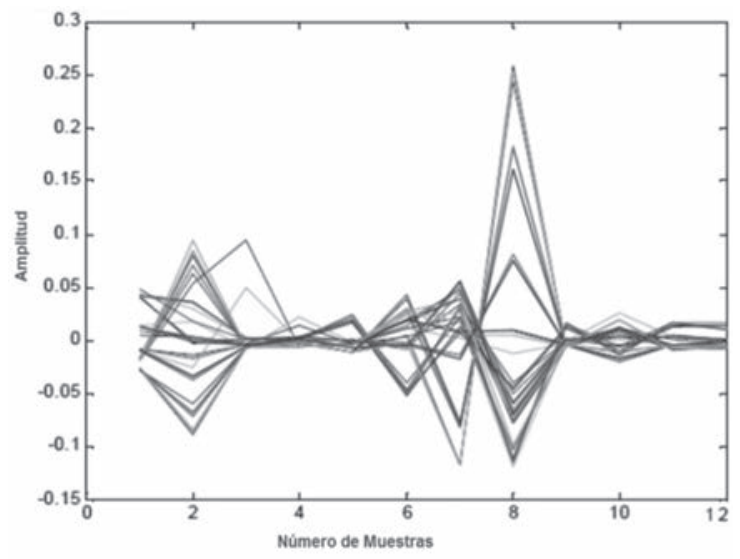

Fuente: Autores del proyecto

\section{3) Extracción característica con Transformada de Fourier}

El análisis de frecuencia es la forma más utilizada para describir las principales características de los registros de actividad eléctrica continua en señales fisiológicas como el patrón por interferencia del electromiograma, aunque también han sido utilizados otros parámetros obtenidos en el dominio del tiempo, como los llamados "turns" y la amplitud total. 
El análisis de frecuencia representa, en realidad, la cantidad de actividad como un continuo en un conjunto de frecuencias.

\section{4) Procesamiento de datos}

Para el procesamiento de cada una de las señales obtenidas por el sistema EMG, depende directamente de los procedimientos realizados durante el pre-procesamiento, de esto obedecerá el éxito en los resultados. Este es el motivo del cuidadoso análisis que se realizó a cada una de las etapas. Las fases que implica el correcto procesamiento de las señales electromiográficas se presentan a continuación:

\section{A. Reducción de la Dimensionalidad}

Cuando se recoge la información de una muestra de datos, lo más frecuente es tomar el mayor número posible de variables. Sin embargo, si tomamos demasiadas variables sobre un conjunto de objetos, por ejemplo 20 variables, tendremos que considerar 180 posibles coeficientes de correlación. Evidentemente, en este caso es difícil visualizar relaciones entre las variables. Otro problema que se presenta es la fuerte correlación que muchas veces se presenta entre las variables: si tomamos demasiadas variables, lo normal es que estén relacionadas o que midan lo mismo bajo distintos puntos de vista. Se hace necesario, pues, reducir el número de variables. Es importante resaltar el hecho de que el concepto de mayor información se relaciona con el de mayor variabilidad o varianza. Cuanto mayor sea la variabilidad de los datos (varianza) se considera que existe mayor información.

\section{B. Análisis PCA}

(Análisis de Componentes Principales)

El método PCA permite reducir, representar y extraer información relevante del conjunto de datos y al mismo tiempo para ver si se pueden determinar agrupaciones ("clúster") espontáneas entre las diferentes medidas realizadas previamente. [9], [10] y [11].

\section{Red perceptron multicapa (MLP)}

La red neuronal MLP está construida usando nodos que se parecen a un sistema de circuitos biológicos neuronales. Se ha demostrado que una red MLP con una capa oculta y la función sigmoidal es un aproximador universal de cualquier función continua en un conjunto compacto. Las redes neuronales MLP pueden trabajar como cualquier clasificador no lineal o como una función de regresión [12], [13] y [14].

\section{Red neuronal probabilística (PNN)}

Esta red es muy utilizada en problemas de clasificación. La red consta de dos capas, una primera red de neuronas de base radial con un número de neuronas igual al número de vectores de entrenamiento, y una capa competitiva de un conjunto de neuronas cuyo número es igual al número de categorías consideradas en el problema de clasificación. Cada neurona de base radial (de la capa de entrada) almacena como pesos uno de los vectores de entrenamiento [15] y [16].

\section{RESULTADOS}

\section{A. Estudio de la señal}

En la Fig. 7 se ilustra la respuesta de una señal electromiográfica sin ningún tipo de procesamiento. Puede apreciarse que aunque es una señal electromiográfica típica no se puede establecer un punto de referencia para su interpretación, es decir, si se desea tomar diferentes muestras se observa que nunca parten de un mismo punto (línea base), por ello la necesidad de realizar un centrado de datos, permitiendo así una mejor visualización y análisis.

Fig. 7. SEÑAL ELECTROMIOGRÁFICA SIN PROCESAR (LÍNEA BASE 3 VOLTIOS DC DE AMPLITUD)

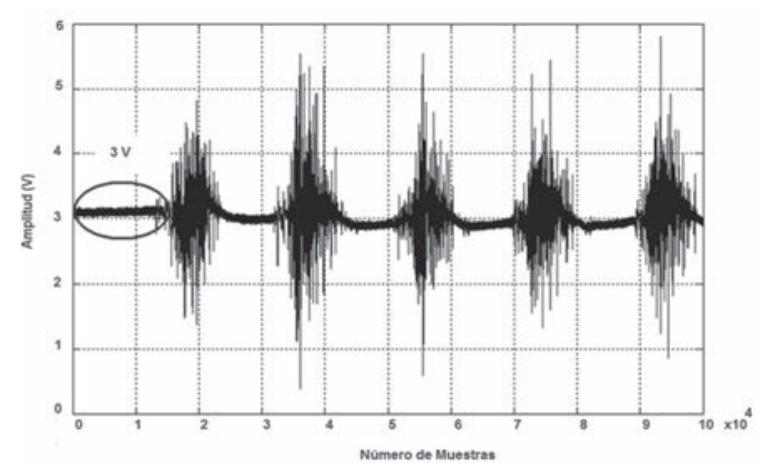

Fuente: Autores del proyecto

En la Fig. 8 se observa que la señal ya tiene un centrado de datos pues es un factor primordial para su posterior interpretación y procesado. 
Fig. 8. SEÑAL ELECTROMIOGRÁFICA APLICADO CENTRADO DE DATOS (LÍNEA BASE APROX. O VOLTIOS DC DE AMPLITUD)

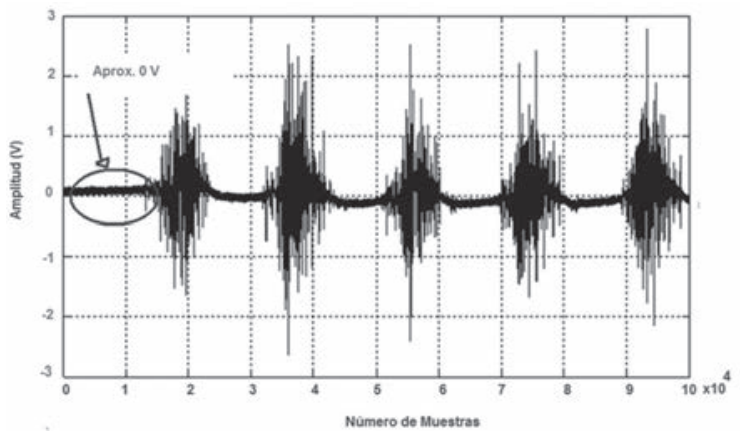

Fuente: Autores del proyecto

Se efectuaron varios registros cuantitativos en contracción isométrica (la longitud del músculo no cambia durante la contracción), durante 4 segundos.

A continuación, tal y como se observa en la Fig.9, se presenta una señal típica electromiográfica de un paciente normal.

Fig 9 SEÑAL ELECTROMIOGRÁFICA PACIENTE SANO

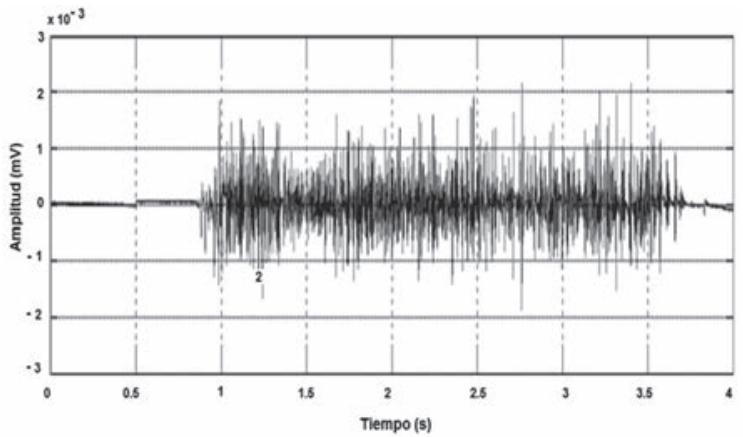

Fuente: Autor del proyecto

En la Fig. 10 se ilustra la respuesta de una señal adquirida en las mismas condiciones pero de un paciente con cuadro clínico pie caído.

Fig. 10 SEÑAL ORIGINAL-CENTRADO DE DATOS

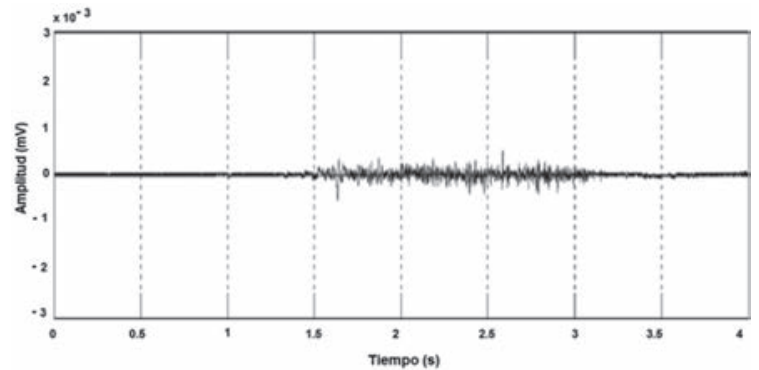

Fuente: Autores del proyecto
Para la interpretación de la Fig. 9 y de la Fig. 10 , se tiene en cuenta las características físicas de la persona. En estos casos las señales se identifican por su forma, es decir, se califica la señal. En éstas se observan tres fases.

- Primera fase: Reposo: el tejido muscular es eléctricamente silencioso. No existen amplitudes significativas.

- Segunda fase: Contracción: cuando el músculo se contrae voluntariamente, la actividad comienza a aparecer. A medida que la contracción aumenta, más y más fibras musculares producen potenciales de acción hasta que aparece un grupo desordenado de dichos potenciales de tasas y amplitudes variables (patrón de interferencia y reclutamiento completo) con la contracción total.

- Tercera fase: Reposo: la señal vuelve a disminuir en amplitud, el músculo se relaja.

Según la interpretación médica se infiere que el paciente presenta un músculo en estado normal (ver Fig. 8), se observó una señal con sus tres fases normales durante la contracción, igualmente los valores de amplitud se encuentran dentro de los estándares (amplitud de $3 \mathrm{mV}$ ).

En la Fig. 9 el paciente presenta un músculo totalmente anormal, anestésico, está desconectado.

Se observó una línea base de valor OV. No presentó reclutamiento, amplitud OV, contracciones nulas. Para este paciente las amplitudes de la señal están por debajo de los $100 \mu \mathrm{V}$, su reclutamiento es muy bajo, lo cual conlleva a inferir un diagnóstico de debilidad muscular.

\section{B. Características Frecuenciales}

Las señales electromiográficas presentan un contenido espectral que puede dar una idea del músculo.

En la Tabla III se describen las características en frecuencia y amplitud de las señales más relevantes tomadas de los pacientes en estudio. 
TABLA III.

CARACTERÍSTICAS EN AMPLITUD Y FRECUENCIA

\begin{tabular}{|c|c|c|}
\hline \multirow{2}{*}{$\begin{array}{c}\text { Paciente } \\
\text { A }\end{array}$} & \multicolumn{2}{|c|}{$\begin{array}{c}\text { Características } \\
\text { Amplitud (mv) } \\
\text { Frecuencia (khz) }\end{array}$} \\
\hline & Máx. pico Amplitud EMG & 1.768 \\
\hline & Máx. pico Amplitud Fourier & 0.7743 \\
\hline & Frecuencia Fourier & 0.164 \\
\hline \multirow[t]{3}{*}{$B$} & Máx. pico Amplitud EMG & 2.137 \\
\hline & Máx. pico Amplitud Fourier & 1.219 \\
\hline & Frecuencia Fourier & 0.291 \\
\hline \multirow[t]{3}{*}{ C } & Máx. pico Amplitud EMG & 2.655 \\
\hline & Máx. pico Amplitud Fourier & 1.18 \\
\hline & Frecuencia Fourier & 0.241 \\
\hline \multirow[t]{3}{*}{$D$} & Máx. pico Amplitud EMG & 2.554 \\
\hline & Máx. pico Amplitud Fourier & 0.7857 \\
\hline & Frecuencia Fourier & 0.663 \\
\hline \multirow[t]{3}{*}{$E$} & Máx. pico Amplitud EMG & 2.368 \\
\hline & Máx. pico Amplitud Fourier & 0.7969 \\
\hline & Frecuencia Fourier & 0.007 \\
\hline \multirow[t]{3}{*}{$F$} & Máx. pico Amplitud EMG & 1.363 \\
\hline & Máx. pico Amplitud Fourier & 0.6925 \\
\hline & Frecuencia Fourier & 0.368 \\
\hline \multirow[t]{3}{*}{ G } & Máx. pico Amplitud EMG & 2.193 \\
\hline & Máx. pico Amplitud Fourier & 0.9502 \\
\hline & Frecuencia Fourier & 0.245 \\
\hline \multirow[t]{3}{*}{1} & Máx. pico Amplitud EMG & 0.01073 \\
\hline & Máx. pico Amplitud Fourier & 0.05394 \\
\hline & Frecuencia Fourier & 0.241 \\
\hline \multirow[t]{3}{*}{2} & Máx. pico Amplitud EMG & 0.2705 \\
\hline & Máx. pico Amplitud Fourier & 0.1007 \\
\hline & Frecuencia Fourier & 0.31 \\
\hline \multirow[t]{3}{*}{3} & Máx. pico Amplitud EMG & 0.7203 \\
\hline & Máx. pico Amplitud Fourier & 0.258 \\
\hline & Frecuencia Fourier & 0.007 \\
\hline \multirow[t]{3}{*}{4} & Máx. pico Amplitud EMG & 0.3623 \\
\hline & Máx. pico Amplitud Fourier & 0.1872 \\
\hline & Frecuencia Fourier & 0.089 \\
\hline \multirow[t]{3}{*}{5} & Máx. pico Amplitud EMG & 0.5155 \\
\hline & Máx. pico Amplitud Fourier & 0.08768 \\
\hline & Frecuencia Fourier & 0.196 \\
\hline
\end{tabular}

Fuente: Autores del proyecto

En las gráficas se presenta un espectro de Fourier, que corresponde a 0.5 segundos de una señal de un paciente sano, (ver Fig. 11) y un paciente con lesión (ver Fig. 12).
Fig. 11 ESPECTRO DE FOURIER, PACIENTE SANO

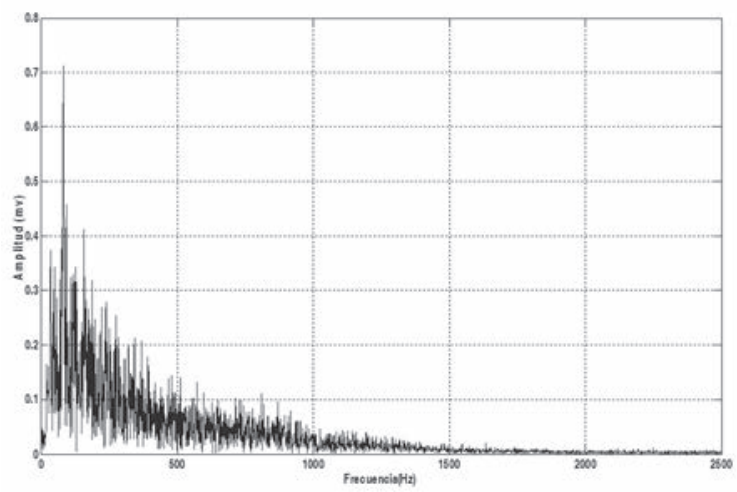

Fuente: Autores del proyecto

Fig. 12 ESPECTRO DE FOURIER, PACIENTE LESIONADO

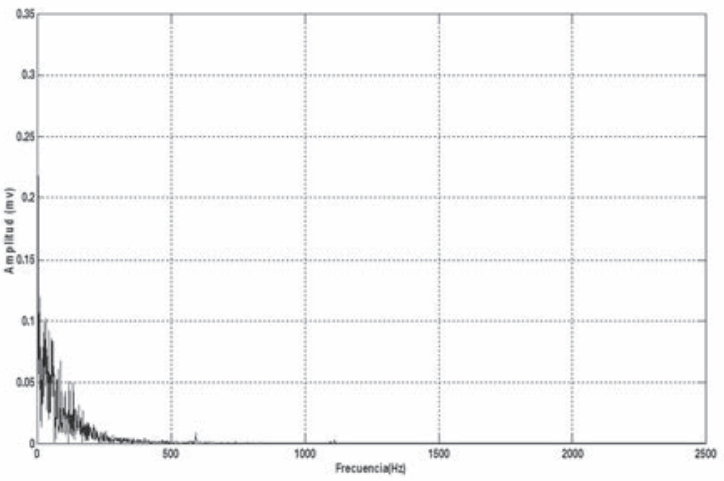

Fuente: Autores del proyecto

Al comparar las características frecuenciales (ver Fig. 13) entre un paciente sano y otro paciente con la lesión, se observa que el espectro del paciente sano (onda con mayor amplitud) posee mayor valor frecuencial y mayor valor de amplitud que el espectro del paciente lesionado (onda menor amplitud); según las gráficas obtenidas del paciente sano (Ver Fig. 11), el mayor valor de frecuencia oscila de 0 a $1000 \mathrm{~Hz}$, mientras que en el paciente con lesión (ver Fig. 12) fluctúa de 0 a $500 \mathrm{~Hz}$.

De estos comportamientos se puede explicar que cuando se realiza una contracción voluntaria, las primeras unidades motoras en activarse son las de contracciones lentas resistentes a la fatiga.

A medida que aumenta la fuerza, se activan las unidades motoras que están compuestas de fibras rápidas resistentes a la fatiga y después se activan las compuestas de fibras de contracción rápida y rápidamente fatigables; dicho de otra forma, a medida que aumenta el nivel de 
fuerza ejercida durante la contracción muscular, se produce un aumento del número de unidades motoras activadas, observable por aumento de la amplitud (reclutamiento espacial) y, por otra, se produce también un aumento de la frecuencia de disparo de las motoneuronas (reclutamiento temporal).

Tales requerimientos condicionan la morfología y las características de las ondas EMG, explicable por los cambios bioquímicos a nivel del músculo, quiere esto decir que el músculo ante un esfuerzo creciente, responde a través de la señal eléctrica de dos formas: a) un aumento de la activación de nuevas fibras musculares, o reclutamiento espacial; b) aumento de la frecuencia de pulsaciones de las unidades motoras o reclutamiento temporal.

Para el espectro del paciente con lesión, se observa que en un principio la amplitud aumenta y hay una disminución de la descarga, con la aparición de una "onda lenta", relacionada de una manera significativa con el comienzo de una sensación de fatiga local, con la sensación de dolor y con la incapacidad del sujeto para mantener la tensión proyectada.

Fig. 13 COMPARACIÓN ESPECTROS DE FOURIER, PACIENTE SANO VS. PACIENTE LESIONADO

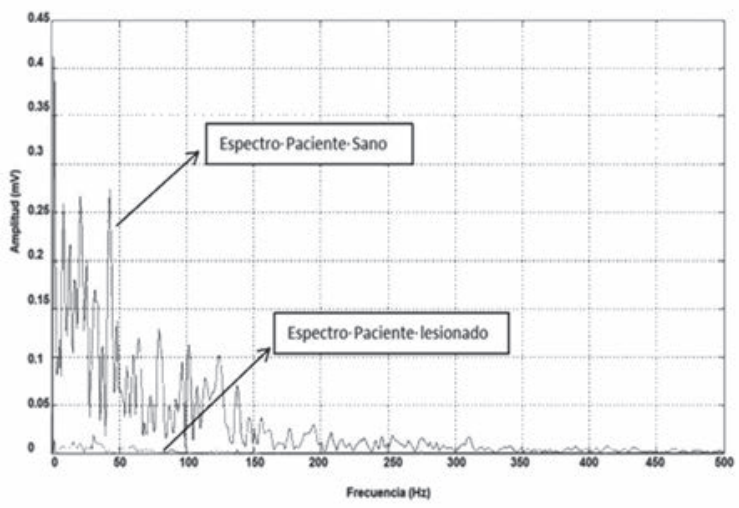

Fuente: Autores del proyecto

Para aplicar el método de PCA se tomó un grupo completo de pacientes sanos y lesionados con el objetivo de obtener un resultado investigativo previo de la respuesta y utilidad que éste puede ofrecer.

En la Fig. 14, se observa un primer resultado, del cual podemos resaltar que se pude distinguir los pacientes sanos de los lesionados, así como las muestras de cada uno.
Fig. 14. RESULTADO ANÁLISIS DE COMPONENTES PRINCIPALES

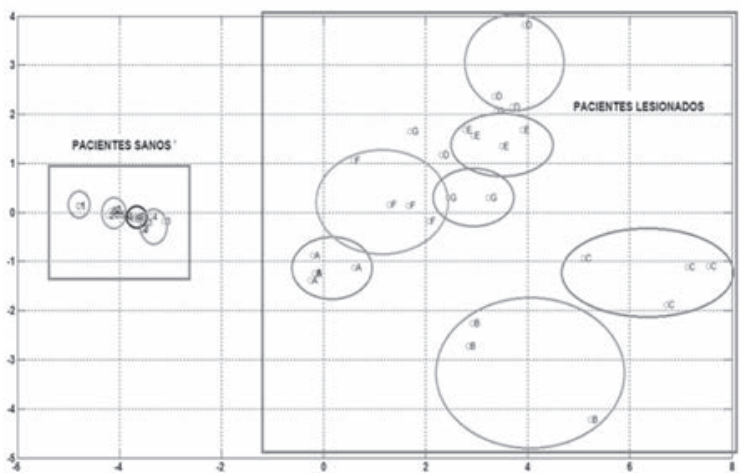

Fuente: Autores del proyecto

En la Fig. 15, se presenta el mismo proceso, pero visto en proyecciones sobre los componentes principales.

Fig. 15. RESULTADO DEL ANÁLISIS DE COMPONENTES PRINCIPALES PROYECCIONES

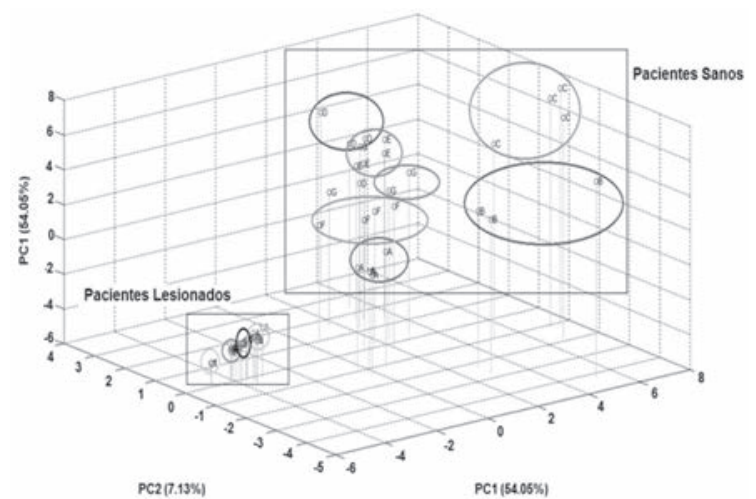

Fuente: Autores del proyecto

Para el caso de señales EMG, PCA es un método útil para la distinción y discriminación de medidas a través de "cluster" de pacientes sanos y pacientes con lesión. Como primer resultado los pacientes que presentaron el cuadro clínico de pie caído se ubican en un solo sector del plano y tienen tendencia a la línea cero, se espera que al realizar un diagnóstico del proceso evolutivo de un paciente éste se ubique más a la izquierda del plano, interpretado una leve mejoría, si es el caso.

Los datos obtenidos del sistema fueron llevados a una hoja de cálculo con el fin de buscar un fundamento matemático que contribuyera a relacionar las variables tratadas. 
En la Fig. 16 se presenta una gráfica en donde se observa los valores numéricos de frecuencia de cada paciente y en la Fig. 17. Se da a conocer respecto a su amplitud.

Fig. 16. VALORES FRECUANCIALES DE CADA PACIENTE

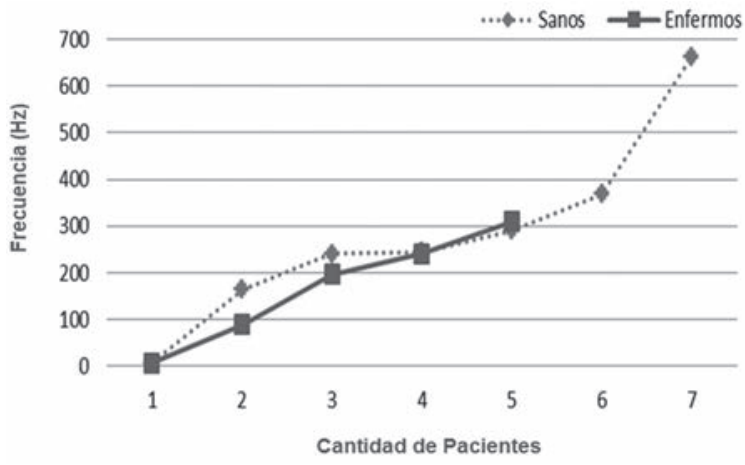

Fuente: Autores del proyecto

Fig. 17. VALORES DE AMPLITUD DE CADA PACIENTE

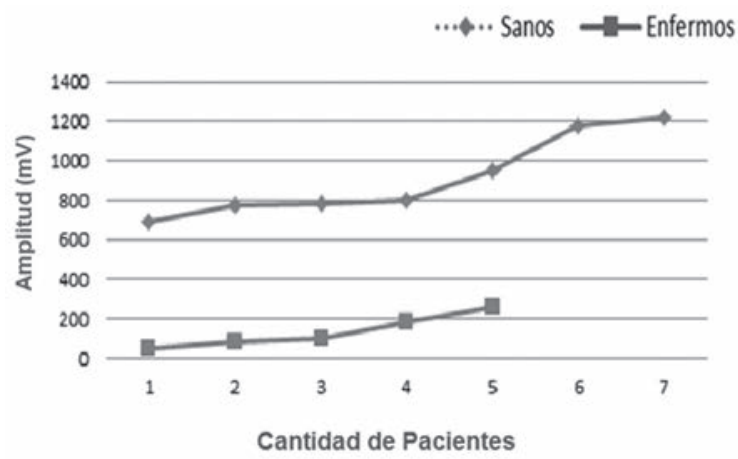

Fuente: Autores del proyecto

Podemos entonces inferir que al realizar un análisis paralelo con respecto a los resultados PCA, efectivamente existe una relación entre la amplitud y frecuencia de la hoja de datos respecto a los resulatdos obtenidos de PCA, ya que las señales se ubican en el plano según sus rangos de amplitud y frecuencia.

\section{Clasificación de medidas}

En la Tabla IV se observa cada una de las respuestas de clasificación del conjunto de 60 medidas y 12 categorías, por medio de redes neuronales, donde se realizaron diferentes agrupaciones con el objetivo de determinar el porcentaje de acierto en la clasificación.
TABLA IV,

CLASIFICACIÓN DE MEDIDAS

\begin{tabular}{|c|c|c|c|}
\hline $\begin{array}{c}\text { Número de } \\
\text { categorías }\end{array}$ & $\begin{array}{c}\text { Caso de } \\
\text { Clasificación }\end{array}$ & $\begin{array}{c}\text { Red } \\
\text { MLP }\end{array}$ & $\begin{array}{c}\text { Red } \\
\text { PNN }\end{array}$ \\
\hline 12 & $\begin{array}{c}\text { Todos los } \\
\text { pacientes }\end{array}$ & $40 \%$ & $80 \%$ \\
\hline 7 & $\begin{array}{c}\text { Pacientes } \\
\text { sanos }\end{array}$ & $60 \%$ & $94,3 \%$ \\
\hline 5 & $\begin{array}{c}\text { Pacientes } \\
\text { lesionados }\end{array}$ & $64 \%$ & $68 \%$ \\
\hline 2 & $\begin{array}{c}\text { Pacientes en } \\
\text { buen estado y } \\
\text { lesionados }\end{array}$ & $100 \%$ & $100 \%$ \\
\hline
\end{tabular}

Fuente: Autores del proyecto

En la mayoría de los casos se obtiene una mejor respuesta con la red PNN.

\section{CONCLUSIONES}

A través de la Transformada de Fourier se logró obtener una característica relevante en el conjunto de datos y en la respuesta de las señales emitidas por los pacientes lesionados y sin lesión.

El empleo de la técnica de reconocimiento PCA demuestra ser una herramienta útil con mayor profundidad y precisión para el estudio de las señales EMG, y además sirve de soporte empírico y metódico qque logra discriminar las muestras de los pacientes saludables con respecto a los afectados. Con la técnica de discriminación PCA, se logró clasificar las muestras de los pacientes según el nivel de complejidad de la enfermedad.

Las redes neuronales son apropiadas para resolver problemas de clasificación, en este caso la red PNN demostró una amplia eficiencia en cuanto a la velocidad de respuesta y sobre todo en el alto porcentaje de acierto en la discriminación de todas las categorías.

El sistema EMG junto con las técnicas expuestas en el presente artículo, demuestra que puede ser utilizado ampliamente y con gran efectividad como herramienta de diagnóstico para identificar enfermedades neuromusculares en las personas.

\section{AGRADECIMIENTOS}

Los autores reconocen y agradecen las contribuciones del grupo de Investigación Sistemas 
Multisensoriales y Reconocimiento de Patrones de la Universidad de Pamplona.

\section{REFERENCIAS}

[1] R.N. Khushaba, S. Kodagoda, M. Takruri, G. Dissanayake, "Toward improved control of prosthetic fingers using surface electromyogram (EMG) signals" Expert Systems with Applications, Vol. 39, Issue 12, pp.10731-10738, September 2012.

[2] K. K. Jung, J. W. Kim, H.K. Lee, S.B. Chung, K.H. Eom, "EMG pattern classification using spectral estimation and neural network" 2007, SICE Annual Conference pp. 1108 - 1111.

[3] T. Doherty, Z. Simmons, B. O'Connel, K. Felice, R. Conwit, K. Ming Chan, T. Komori, T. Brown, D. Stashuk, and W. Brown "Methods for Estimating the Numbers of Motor Units in Human Muscles", 1995, Journal of Clinical Neurophysiology, Vol.12, pp.:565-84.

[4] A. Hamilton-Wright, D.W. Stashuk, "Physiologically based simulation of clinical EMG Signals", 2005, Biomedical Engineering, IEEE Transactions on, Vol.: 52, Issue: 2, pp. 171 - 183.

[5] G. Cometti, Métodos modernos de musculación, Editorial Paidotribo, cuarta edición, 2005.

[6] C. Durán, A. Jaimes, "Diseño de un registrador electromiográfico de superficie para cuantificar unidades motoras en la enfermedad del pie Caído" Revista: Revista Colombiana de Tecnologías de Avanzada, Vol 2, No. 1, pp. 57 - 62, 2010.

[7] Normativa SENIAM (Surface ElectroMyoGraphy for the Non-Invasive Assessment of Muscles, online, Available, http://www.seniam. org//.

[8] M. Reaz, M. Hussain, F. Mohd-Yasin. “Techniques of EMG signal analysis; Detection, processing, classification and applications", Biological Procedures, Vol.8, 1, pp.163, 2006.
[9] D. R. Rogers, T. Dawn. Isaac. M, "EMG-based muscle fatigue assessment during dynamic contractions using principal component analysis" Journal of Electromyography and Kinesiology, Vol. 21, Issue 5, pp.811-818, October 2011.

[10] K. Kiatpanichagij, N. Afzulpurkar, "Use of supervised discretization with PCA in wavelet packet transformation-based surface electromyogram classification", 2009, Biomedical Signal Processing and Control, Vol. 4, Issue 2 pp.127-138, April 2009.

[11] S.Park, J.H.Na, J.Y.Choi, "PCA-based feature extraction using class information Myoung, Systems", Man and Cybernetics, 2005 IEEE International Conference on, Vol.: 1, pp. 341 - 345.

[12] I.B.V. Da Silva, P.J.L, Adeodato, "PCA and Gaussian noise in MLP neural network training improve generalization in problems with small and unbalanced data sets" 2011, Neural Networks (IJCNN), The 2011 International Joint Conference , pp. $2664-2669$.

[13] W. Yu, H. Yamaguchi, H.Yokoi, M. Maruishi, Y.Mano, Y.Kakazu, "EMG automatic switch for FES control for hemiplegics using artificial neural network", Robotics and Autonomous Systems, Vol. 40, Issues 2-3, pp. 213-224, 31 Aug 2002.

[14] Y.I Özbay, G. Tezel," A new method for classification of ECG arrhythmias using neural network with adaptive activation function", Digital Signal Processing, Vol. 20, Issue 4, pp. 1040-1049, July 2010.

[15] K. Liang, W.Chen. "Fast adaptive PNN-based thresholding algorithms, Pattern Recognition", Vol. 36, Issue 12, pp. 2793-2804, December 2003.

[16] H.C.Dubey, Nandita, A.K. Tiwari, "Blind modulation classification based on MLP and PNN, 2012 in Engineering and Systems (SCES), Students Conference, pp: 1-6. 